\title{
DIVERSIDAD, IGUALDAD, CULTURA ESCOLAR: SIGNIFICADO E IMPLICACIONES PRÁCTICAS EN LA ENSEÑANZA SECUNDARIA OBLIGATORIA
}

\author{
DIVERSITY, EQUITY, SCHOOL CULTURE: MEANING AND IMPLICATIONS \\ FOR PRACTICE AT THE COMPULSORY SECONDARY EDUCATION LEVEL
}

\author{
Teresa Aguado* \\ Universidad Nacional de Educación a Distancia
}

\begin{abstract}
RESUMEN
Tres son las dimensiones en torno a las cuales se articula nuestra reflexión en relación con la atención a la diversidad: el desarrollo de nuevos significados sobre qué sea la diversidad, la modulación de una cultura escolar que acepte la diversidad, y la promoción de prácticas inclusivas que aseguren el éxito escolar y eviten la discriminación de determinados grupos de estudiantes. Esto supone actuar sobre dimensiones tradicionalmente escasamente atendidas, como son: la revisión de los criterios y procedimientos de diagnóstico aplicados habitualmente para clasificar, seleccionar y evaluar a los estudiantes, y las conexiones del centro con la comunidad.
\end{abstract}

Palabras clave: diversidad del alumnado, igualdad de oportunidades, educación intercultural, prácticas escolares inclusivas.

\begin{abstract}
Secondary Schools in Spain are serving a more heterogeneous student population now than ever before. This article explores the role of school in responding the needs of diverse students. Inclusive school culture is rooted in values of equity and social justice. Three dimensions are highligted: fostering new meanings about diversity, promoting inclusive school cultures and instructional programmes; to review the assessment criteria and processes and building relationships between schools and communities.
\end{abstract}

Key words: students diversity, opportunity equity, intercultural education, inclusive practices at school.

* Profesora titular de Pedagogía Diferencial. Departamento M.I.D.E. de la UNED. Líneas de investigación: diversidad cultural en contextos educativos. 


\section{Diversidad, igualdad, cultura escolar}

La Reforma del Sistema Educativo se estableció a partir de unos principios básicos, entre otros, el de la mejora de la calidad de la enseñanza a partir de un currículum integrador atendiendo a la diversidad de los alumnos. La Educación Secundaria Obligatoria se diferencia de etapas educativas anteriores, a las que sustituye, por su condición de etapa obligatoria y, por tanto, accesible a todo el alumnado; por tener que ajustarse a los intereses del alumnado; por la exigencia de impartir conocimientos adaptados de forma que sean asimilables para conseguir los objetivos de la etapa; porque persigue la formación de ciudadanos capaces de desenvolverse en una sociedad democrática y plural.

La atención a la diversidad del alumnado y el logro de una igualdad de oportunidades real está siendo un reto permanente para los profesionales que ejercen su trabajo en esta etapa de la secundaria obligatoria. El sistema educativo, los agentes, profesionales e instituciones que lo conforman, no están siendo capaces, en la mayoría de los casos, de introducir un cambio que vaya más allá de medidas aisladas y que afecte a la cultura misma vivida y transmitida por la educación. Se propone un cambio en la cultura escolar, un cambio que pasa por revisar el concepto mismo de diversidad y que considera la heterogeneidad como norma. A partir de ahí, es posible responder a la cuestión permanente de decidir cómo organizar el trabajo de forma adecuada a un alumnado que es diverso por razón de etnia, raza, clase social, género, origen nacional, lengua materna, orientación sexual, habilidades físicas.

El reconocimiento y la atención a las diferencias de los alumnos y de sus comunidades es condición inexcusable de toda etapa de enseñanza obligatoria tanto por razones axiológicas derivadas de los principios asumidos por sociedades que, como la nuestra, defienden derechos de igualdad, equidad y participación social, como porque es la garantía que permite alcanzar objetivos educativos esenciales, como son la construcción de la propia identidad y la igualdad de oportunidades en el acceso a todos a los bienes y recursos socioeducativos disponibles. La articulación de diversidad e igualdad en educación implica tener en cuenta que:

a) La educación formal es la vía principal para lograr el éxito o tener una vida digna y no hay disponible otro camino; es preciso investigar acerca de las razones que explican las diferencias en cuanto a fracaso/éxito escolar entre los grupos de alumnos establecidos en función de características diferenciales, para actuar sobre ellos.

b) Los alumnos, sus familias y comunidades de referencia son diferentes y desiguales. Los rasgos personales y sociales que no están en armonía con el «patrón» escolar ni con las exigencias del mercado de trabajo son erradicados y erosionados. Su mantenimiento actúa como lastre para lograr el éxito en la escuela.

c) La cultura transmitida por la escuela es elaborada desde un determinado patrón sociocultural de referencia, los alumnos pertenecientes a dicho grupo «juegan con ventaja» en el acceso y el uso de los recursos escolares frente a los alumnos más alejados de ese patrón de referencia.

d) Determinadas prácticas escolares mantienen, acentúan y legitiman las desigualdades sociales de los alumnos a base de no reconocer y valorar sus diferencias. Frente a esto debería ser posible una escuela igual y eficaz cuya influencia supere las diferencias iniciales de los alumnos provenientes de grupos socioculturales diferentes. 
e) La igualdad de oportunidades debería entenderse como una cuestión de posibilidad de elección, la cual está determinada por la interacción entre características individuales y condiciones sociales. La forma en que las instituciones manejan estas relaciones, en orden a lograr resultados educativos, determina las opciones reales de los alumnos y conforma sus carreras académicas. Garantizar la igualdad de oportunidades supone proporcionar fórmulas diferentes para personas diferentes.

Tres son las dimensiones en torno a las cuales se va a centrar nuestra reflexión en relación con la atención a la diversidad: el desarrollo de nuevos significados sobre qué sea la diversidad, la modulación de una cultura escolar que acepte y apoye la diversidad, y la promoción de prácticas inclusivas que aseguren el éxito escolar y eviten la discriminación de determinados grupos de estudiantes.

\section{Nuevos significados sobre qué es la diversidad}

La diferencia puede ser presentada como complementariedad o como asimetría y desajuste entre individuos y grupos. En el ámbito educativo son diversas las respuestas y soluciones que se han propuesto según el valor otorgado a las diferencias y los enfoques desde los que se explican: de déficit, diferenciales, integradores, adaptativos, dinámicos. Dichos enfoques se configuran a partir de determinados presupuestos ideológicos, surgen y perviven en relación a determinadas concepciones sobre qué es ser persona y qué tipo de sociedad es la deseable (Aguado, 1995).

Los modelos de déficit entienden las diferencias como alejamiento e inconsistencia con respecto a pretendidos modelos ideales. Hay una forma ideal de ser alumno, de ser profesor, de ser ciudadano, ... Lo diferente se valora negativamente y se aspira a igualar, surgen los programas compensatorios e integradores, de minorías, de marginados, de «especiales», ... Los modelos diferenciales, por su parte, enfatizan el valor de la diversidad, analizan las diferencias evitando implicaciones negativas y la comparación con supuestos patrones ideales. La diferencia se considera un constructo dinámico y cambiante, y se coloca en el centro del discurso educativo.

El desarrollo de estructuras y prácticas de atención a la diversidad debe ir acompañado de la asunción de nuevos valores; si esto no es así, no se producirán cambios reales ni duraderos. La difusión de unos significados y creencias sobre la diversidad y la forma de abordarla implica algo más que la simple comunicación o intercambio entre los participantes de sus ideas y convicciones. Supone básicamente la generación de nuevos significados en el contexto mismo en que se sitúe la acción educativa.

En todo centro educativo, en todo claustro de profesores, en todo equipo de ciclo, o de orientadores, en cualquier decisión educativa, están presentes implícita y explícitamente un complejo agregado de significados, creencias y valores que se legitiman mediante su aceptación por parte de un público más amplio y que se codifican a través de la estructura del centro, su cultura, y las rutinas diarias (normas, temporalización, organización del espacio, secuenciación de tareas, distribución de la autoridad, etc.). Los centros escolares se construyen, de hecho, alrededor de lo que la gente piensa sobre ellos, de los significados y el sentido que se les atribuye. Los cambios organizativos reales se producen no simplemente cuando se abordan cambios técnicos en la estructura y el proceso, sino cuando las personas, den- 
tro y fuera de la institución, construyen y manejan nuevos significados a lo que consideran como «cambio».

Los grupos y los individuos no somos simples receptores sino creadores, generadores de significados. Es preciso plantearse cómo las prácticas y representaciones de la diferencia son aprendidas, interiorizadas y transformadas activamente en el proceso de educar (Giroux y Mc Laren, 1989), entendiendo éste como interacción entre personas en un contexto determinado que conduce a la producción de conceptos y leyes de conocimiento, interpretaciones específicas de la realidad. La diferenciación llevada a la práctica es ante todo una dinámica y no un sistema; las diferencias son constructos dinámicos, cambiantes y no etiquetas inamovibles (Skutnabb-Kangas, 1988).

Una estrategia clara disponible para revisar las visiones mismas de la diversidad y la igualdad presentes en un determinado contexto educativo es la de promover el discurso democrático entre la comunidad escolar. Son muchos los autores que, tanto desde tradiciones normativas y críticas como desde perspectivas empíricas, han explorado cómo los procesos de discurso democrático en las escuelas pueden generar prácticas educativas que sirven a las necesidades de los estudiantes diversos (Rusch, 1998).

Sin embargo, los estudios previos y nuestra propia experiencia permiten afirmar que el discurso democrático transformador no está a menudo presente en nuestros centros educativos, ni siquiera en los centros públicos. En su lugar, es mucho más común la asunción de formas más o menos explícitas de discurso liberal. ¿Por qué esta huida, esta reacción elusiva ante el discurso democrático y la igualdad de oportunidades real para todos?

Las razones que encontramos son varias. Primero, la escuela refleja las formas de relaciones sociales que se dan en el contexto social al que sirve, y se mimetiza con los planteamientos homogeneizadores y asimilacionistas que no reconocen las diferencias culturales y sociales que caracterizan a sus miembros (Corson, 1995). Por ejemplo, entre los propios profesores se producen la anulación de las diferencias de clase, intereses, medio cultural; llegar a ser profesor implica para muchos la negación o la crítica de sus culturas o referentes axiológicos previos, y esta negación aparece como la única vía posible para ser un buen profesor. Se produce un fenómeno de extrañamiento respecto a lo diverso y a sus propias cosmovisiones y códigos (verbales y no verbales). La consecuencia es que esto se reproduce en el aula, en el claustro, en las salas de profesores y se anula toda posibilidad de discurso democrático inclusivo.

Segundo, el discurso democrático en torno a la diversidad no está ligado de forma autocrítica a cuestiones de justicia social e igualdad. Tres, la democracia tiene sus propios peligros de coacción (Foucault, 1980; Hargreaves, 1994). Y cuarto, es a menudo difícil darse cuenta, ser consciente de hasta qué punto todas las decisiones que se adoptan en el proceso escolar afectan al carácter inclusivo o no de la acción educativa. Por ejemplo, una forma frecuente de convertir a la diversidad en invisible es no conocer cuáles son los patrones demográficos de la comunidad a la que el centro atiende, de ignorar datos fundamentales sobre experiencia escolar previa, condiciones de vida, origen nacional, código lingüístico, ... Otra, apoyada incluso por la administración y la política educativa al uso, es establecer prácticas de atención a la diversidad sin conexión con el programa curricular central de la escuela. Este es el caso de las medidas compensatorias, los cursos «suplementarios» de español para extranjeros, las aulas-taller, las aulas de «recuperación», los programas de garantía social, etc. (Capper, 1993; Lluch y Salinas, 1996; Aguado, 1999). 


\section{Por una cultura escolar inclusiva}

Más allá de la mejora de la instrucción, el enfoque que nos interesa a la hora de desarrollar medios escolares que sirvan a las necesidades derivadas de la diversidad del alumnado, es el que propone la elaboración de culturas escolares que sean inclusivas respecto a las distintas manifestaciones de la diversidad.

En esta perspectiva se sitúan las propuestas denominadas multiculturales o interculturales. Así, Baptiste (1999) define «multiculturalismo» como,

... una reforma filosófica y comprensiva del medio escolar centrada esencialmente en los principios de igualdad, éxito, y justicia social para todos los estudiantes. La igualdad es el resultado de modificar el medio escolar, especialmente los componentes instructivos y curriculares, organizativos y estructurales, de forma que los estudiantes de diversos grupos raciales, étnicos y sociales vivan experiencias escolares que favorezcan la igualdad y el éxito. El éxito se estimará atendiendo a la representación paritaria de todos los grupos (sociales, culturales, étnicos, de género) en las medidas del rendimiento académico que se adopten. (p. 107)

Por su parte, en nuestro contexto, lo intercultural en educación ha sido definido por la autora de este artículo (Aguado, 1995) como:

«Enfoque educativo basado en el respeto y valoración de la diversidad cultural, dirigido a todos y cada uno de los miembros de la sociedad en su conjunto que propone un modelo de intervención, formal e informal, holístico, integrado, configurador de todas las dimensiones del proceso educativo en orden a lograr la igualdad de oportunidades/resultados, la superación del racismo en sus diversas manifestaciones, la comunicación y competencia interculturales» (Aguado, 1995).

Los presupuestos pedagógicos desde los que se fundamenta el enfoque adoptado se concretan en dos:

1. Modelo diferencial-adaptativo que define las características de individuos y grupos como relaciones dinámicas y no como entes fijos utilizados para etiquetar y clasificar. Las dificultades académicas de grupos determinados no pueden explicarse/justificarse en términos de discapacidad del alumno o el grupo por supuestas deficiencias genéticas (Coleman, 1966) o por carencias socioculturales (Cohen y cols. 1968).

2. El análisis realizado desde la pedagogía crítica al insistir en la necesidad de promover la mejora colectiva y la igualdad estructural en el seno de la sociedad multicultural. Se señala la restricción e inadecuación de conceptos basados en la promoción individual dentro de una organización social injusta y perpetuadora de diferencias sociales.

Las bases teóricas aquí formuladas son el entramado conceptual en el que se inserta el enfoque de atención educativa a la diversidad aquí propuesto enunciado, del que se derivan algunos principios fundamentales para la práctica y la investigación en el ámbito escolar (Hardgreaves, 1994; Banks, 1996):

1. La meta última a lograr es la reforma de la escuela y otras instituciones educativas que permitan a los alumnos de diversos grupos raciales, culturales y sociales experimentar una educación equitativa en igualdad de oportunidades (de entrada y de salida del sistema educativo). 
2. Los cambios en el sistema educativo deben incluir cambios no sólo en el currículum, sino en todas las dimensiones del proceso: actitudes y formación del profesorado, estrategias de enseñanza, motivación y comunicación, materiales y recursos, agrupamientos y evaluaciones, metas y normas del centro.

3. Atender a la integración de contenidos y a los procesos mediante los que se construye el conocimiento. Lo primero implica el uso de ejemplos, datos, informaciones de los diversos grupos y culturas con los que ilustrar conceptos básicos, principios, generalizaciones y teorías. Lo segundo supone considerar la forma en que las asunciones culturales implícitas, los marcos de referencia, perspectivas y sesgos dentro de una disciplina influyen en las formas en que el conocimiento se construye.

4. La educación recibida en las escuelas debe garantizar no sólo la igualdad de oportunidades de acceso a la educación, sino la igualdad de experiencias eficientes y potenciadoras. Esto implica la utilización de técnicas y métodos que faciliten el éxito académico de los alumnos de diversos grupos sociales, raciales y culturales.

5. La superación del racismo y la discriminación exige el análisis de las actitudes raciales de alumnos y profesores para sugerir estrategias que ayuden a desarrollar actitudes y valores democráticos y el desarrollo de la competencia para desenvolverse en medios socioculturales diversos.

Desde estas recomendaciones básicas, varias son las propuestas que se derivan para una práctica inclusiva que atienda a las necesidades de un alumnado diverso (Parker y Shapiro, 1993; Miron, 1997; Katz, 1999; Walker, 1999):

- Adoptar estrategias didácticas personalizadas y tratar a los estudiantes como individuos más que como representantes de un determinado grupo o categoría. Valorar el saber cultural que los estudiantes aportan y utilizarlo como parte de la cultura transmitida, ayudando a comunicarla y compartirla.

- Reconocer el conflicto étnico cuando se manifieste y utilizarlo como una oportunidad para introducir cambios positivos Crear un clima muy cuidado en el que sea posible la cooperación entre padres, profesores, estudiantes y comunidades.

- Mantener altas expectativas respecto a todos los estudiantes.

- Centrarse en el logro de altos resultados académicos y disponer de adecuados apoyos materiales y humanos accesibles a todos.

- Configurar la estructura escolar de forma que se garantice el acceso igual y efectivo de todos a la instrucción.

- Estimular a los profesores a que analicen sus prácticas diarias en orden a detectar posibles sesgos y discriminaciones en función del origen social y cultural de sus alumnos. Adoptar las medidas necesarias para mantener la comunicación con las familias mediante visitas a domicilio, uso de mediadores y traductores, desarrollar competencias en los padres para apoyar el aprendizaje de sus hijos.

\section{Prácticas inclusivas en el interior de la escuela}

La mayor parte de lo escrito en relación con la reforma de la escuela y la atención a la diversidad se deriva y está directamente vinculado a procesos de formación (Pérez, 1990) y 
técnicos (Wang, 1995), especialmente referidos a procesos de enseñanza y aprendizaje. Se han propuesto nuevos o renovados métodos instructivos, tales como los basados en proyectos o los formulados desde enfoques constructivistas. Se formulan nuevas propuestas organizativas, tales como los centros más pequeños, los grupos reducidos y flexibles, los horarios sin rígidas divisiones por asignaturas. Se plantean nuevas formas de desarrollo profesional basadas en la colaboración y el intercambio.

Todos estos son ejemplos de iniciativas contempladas en las propuestas de reforma orientadas a dar una mejor respuesta a las necesidades derivadas de la diversidad del alumnado. En muchos casos se han realizado esfuerzos, más o menos persistentes y recurrentes, para evaluar su utilidad en orden a mejorar las experiencias educativas y los resultados obtenidos por grupos diversos de estudiantes. Sin embargo, la reforma, para ser efectiva, debe implicar, como ya se ha dicho, el desarrollo de una cultura escolar inclusiva. Esto supone actuar sobre dimensiones tradicionalmente escasamente atendidas, como son las dos que a continuación se analizan: la revisión de los criterios y procedimientos de diagnóstico aplicados habitualmente para clasificar, seleccionar y evaluar a los estudiantes, y las conexiones del centro con la comunidad.

\section{Diagnóstico y evaluación}

Decisiva en el logro de acciones educativas eficaces es la atención dedicada al estudio de las variables implicadas en el proceso de diagnóstico y evaluación educativos. Los profesores y orientadores, de forma mayoritaria, carecen de preparación específica y raramente se cuestionan el significado mismo de diversidad, es decir, cuál es el supuesto patrón ideal que están midiendo. Las investigaciones realizadas han mostrado la gran importancia de variables como son la sensibilidad del orientador y las estrategias utilizadas en el proceso orientador en el logro de los objetivos propios de modelos de atención a la diversidad.

Las pruebas y técnicas de diagnóstico presentan un alto componente verbal y seleccionan atendiendo casi exclusivamente a aspectos en los que los grupos minoritarios y socialmente deprivados presentan niveles más bajos. Generalmente, se asocia déficit lingüístico con déficit cognitivo y con frecuencia se realiza un diagnóstico subjetivo de las capacidades de los estudiantes utilizando como indicadores la apariencia física, la conducta de relación con los demás, el lenguaje y una combinación de factores sociales que incluyen ingresos, nivel educativo y tamaño familiar, número de hermanos, servicios sociales recibidos (Jackson y Cosca, 1974). Así, sucede que los alumnos de clases sociales más bajas o de grupos culturales minoritarios son adscritos a clases de recuperación o niveles inferiores a los de sus capacidades reales con la consiguiente discriminación en cuanto al tratamiento educativo. Esto provoca la acentuación de las diferencias con respecto a los alumnos del grupo mayoritario y la confirmación de las expectativas generadas respecto a las diferencias entre grupos (Samuda y Crawford, 1986).

Las prácticas habituales de diagnóstico y evaluación adolecen de un sesgo discriminatorio más o menos encubierto dado que plantean un patrón único independientemente de la clase o grupo de origen, basan su credibilidad en los índices de correlación y validez que justifique etiquetados y jerarquización, y atribuyen la inferioridad mental a la pertenencia a determinados grupos. Los tests habitualmente utilizados, referidos a una norma, tienen en cuenta el producto, no el proceso, de forma que no ayudan a reestructurar la enseñanza en 
orden a optimizar las cualidades del alumno y profesores ni responden a sus reales necesidades educativas.

Los procedimientos de diagnóstico y evaluación deberían tener en cuenta la evidencia aportada por las investigaciones sobre interacciones entre variables afectivas y culturales relativa a variables no cognitivas que son relevantes en los resultados educativos de estudiantes pertenecientes a diferentes grupos socioculturales: variables que no están entre las tradicionalmente asociadas al éxito académico y que son (Rosenthal y Jakobson, 1968; Chaikin y Derlega, 1978; Nieto, 1992):

a) Autoconcepto positivo individual y en relación con la valoración de su propia identidad como grupo cultural específico.

b) Comprensión y capacidad para manejar la discriminación y marginalidad.

c) Autovaloración realista. Son conscientes de sus limitaciones y capacidades.

d) En general, los estudiantes de grupos minoritarios presentan un locus de control externo, no perciben sus éxitos y fracasos como responsabilidad propia sino debidos a razones, causas ajenas a ellos mismos. No perciben que su esfuerzo suponga mejores resultados.

e) Motivación para la ejecución y control percibido sobre sus vidas: metas a largo plazo derivadas de elecciones propias.

f) Familia con altas expectativas respecto a sus resultados. Generalmente existe una persona adulta que ejerce o ha ejercido fuerte influencia en su entorno y actúa como modelo a seguir.

g) Sentido de pertenencia al grupo y pensamiento crítico.

h) Vinculación a la comunidad de origen y participación en actividades extraescolares. Experiencia de liderazgo.

i) Conocimientos e intereses variados, más allá de lo académico.

Estos resultados fueron ampliamente corroborados en una investigación reciente en la que se estableció el perfil de alumnos gitanos con éxito en la escolaridad primaria y que accedían a la Enseñanza Secundaria Obligatoria. (Aguado y cols., 1998). En el caso de estos alumnos, todos ellos escolarizados en centros públicos de diferentes Comunidades Autónomas, se añadía otra variable propia: todos eran chicos, ninguna chica; como evidencia de la fuerte discriminación escolar derivada de algunas características asociadas al grupo étnico.

La identificación con el propio grupo de referencia tiene importantes funciones psicológicas y debería fomentarse sin caer en el error de creer que debe ser considerada como una cultura separada, ajena a la oficial o escolar. Hemos de tener presente que la autoafirmación como grupo, si bien no es un proceso uniforme en todo el mundo, se relaciona con la defensa de intereses económicos y sociales. Las personas aceptan, rechazan y modifican su identidad social si supone un beneficio o ventaja para ellos. Según esta metáfora de la propiedad cultural, la identidad se presenta como una cualidad opcional, casi voluntaria, a menudo más simbólica que real si uno considera la identidad cultural aisladamente en términos de tradición cultural auténtica. El autoconcepto positivo se relaciona con intereses psicológicos, sociales y económicos; y condiciona las expectativas generadas en torno al éxito académico. 
Por lo que se refiere a la motivación y control percibido, la teoría de la atribución señala que la gente trata de explicar los acontecimientos que ocurren en sus vidas acudiendo a factores internos o externos más o menos estables (Weiner, 1972). Parece darse una relación entre el grado de motivación y el nivel de control que percibimos sobre nuestras acciones. Diferentes grupos pueden tener diferentes percepciones de la controlabilidad de sus resultados académicos, lo cual modula su actitud, expectativas y respuestas hacia la escuela en la medida en que afectan su grado de concentración, persistencia en la tarea, esfuerzo y estrategias utilizadas en el aprendizaje. El reto que queda planteado a toda propuesta de educación atenta a la diversidad de los alumnos es cómo fomentar una identidad personal/social fuerte, reconociendo una variedad de intereses diferentes, estilos culturales y la necesidad de todos los individuos y grupos a mantener un cierto grado de autoestima, dignidad y orgullo para poder obtener beneficios de la experiencia académica que se les ofrece.

\section{Construir conexiones entre el centro y la comunidad}

A medida que se reconoce la complejidad de las necesidades de los estudiantes y de sus comunidades de referencia, se hace más evidente que la escuela no puede funcionar como un ente aislado, ajeno al entramado social que le rodea. La descripción del medio social en el que se está inserto pasa por reconocer el entramado de grupos, asociaciones e instituciones que la acompañan. El centro educativo ocupa una posición privilegiada como agente promotor y canalizador de iniciativas que favorezcan la vinculación del sistema educativo general con intervenciones propias de la educación no formal. Las fórmulas para articular la vinculación entre centro, familia y comunidad son varias, destacamos dos: colaboración y participación de las familias e implicación del centro en las iniciativas de la comunidad (Aguado, 1995).

La escuela debería situarse como parte de una red de instituciones en la que se inserta y de la que participan sus estudiantes. Se trata de sacar partido de los recursos disponibles y proporcionar servicios coordinados que atiendan las necesidades de los estudiantes y sus comunidades. Esto implica que el medio escolar se movilice en el desarrollo comunitario y trabaje con otras organizaciones poniendo a disposición de los jóvenes sus instalaciones y recursos. Son ejemplos frecuentes de instituciones de esta red potencial: familias, iglesias, asociaciones de voluntarios, movimientos vecinales, etc. Hay escasa evidencia acerca de la relación entre la escuela y la comunidad, así como sus implicaciones educativas. La mayor parte de los autores asumen que los estudiantes diversos en dimensiones como son la raza, etnia, clase social, origen nacional, dificultades físicas, tienen más necesidades de servicios ofrecidos por organizaciones diversas que otros alumnos. Son problemas frecuentes la escasa oferta de servicios específicos y la fragmentación de los que hay disponibles (Massell, 1990).

La segunda línea de actuación se refiere a la implicación de los alumnos y sus familias en la elaboración de las normas que regulan la conducta y la disciplina. Los estudiantes de culturas distintas a la mayoritaria perciben que sus tradiciones, lenguaje y experiencias vitales están excluidas de la escuela. Los profesores no disponen de tiempo para planificar, colaborar y formarse. Es evidente la escasa implicación de los padres y ausencia de lazos con la comunidad. Sin embargo, la colaboración con las familias proporciona la más segura y relevante información que sirva para atender las necesidades individuales. Lograrla implica 
establecer comunicación escrita que proporcione datos sobre el centro, los programas, las decisiones y normas; mantener contacto individual con los padres mediante entrevistas, visitas al hogar; asegurar la participación de todos los grupos en los órganos de gobierno; recurrir a la formación de mediadores y traductores, etc.

Un ejemplo de este último tipo de iniciativas lo constituyen los programas de formación de mediadores socioculturales que sirvan de catalizadores en las relaciones entre el medio educativo, la familia y la comunidad (EMSI, 2000). Estos programas se centran en el desarrollo de competencias comunicativas y de intercambio entre grupos diversos. Se centran en actividades que promueven las relaciones interpersonales, el desarrollo de actividades más allá del medio académico, el adecuado diagnóstico de necesidades, el acceso a recursos comunitarios, las interacciones entre familias, profesores y alumnos.

Los estudios realizados ponen de manifiesto los efectos positivos que la participación de los grupos considerados diversos o minoritarios en el gobierno de la escuela -a través de mecanismos de participación externa e interna- tiene tanto sobre la comunidad y la familia como sobre el aprendizaje de los alumnos minoritarios. A partir de este análisis y desde una perspectiva crítica, han surgido iniciativas «antideficitarias» como las de los «padres igualadores», las «escuelas aceleradas» y las escuelas alternativas de Deborah Meyer. Su objetivo no es remediar, sino acelerar el aprendizaje y el desarrollo, tomar en serio la idea de democracia cultural y política de la diferencia (Giroux, 1991). Se asume la necesidad de implicar a todos en el proceso educativo, no sólo como espectadores receptores de información, sino como agentes activos que comparten significados, negocian, establecen y evalúan los logros.

\section{Consideraciones finales}

Como ya se ha dicho, la enseñanza secundaria obligatoria atiende a un alumnado diverso en función de categorías que van más allá de las diferencias tradicionalmente asumidas. Es preciso tener en cuenta las diferencias de los alumnos en función de la clase social, el origen étnico, el medio profesional de la familia, el género y la orientación sexual, la lengua, entre otros. Todas estas diferencias han estado siempre presentes en el alumnado de enseñanza primaria y se han hecho presentes por primera vez en secundaria gracias a la reforma educativa. Los profesionales que trabajan en esta etapa educativa deben afrontar el reto de dar respuesta a las nuevas exigencias surgidas de un alumnado que se reconoce diverso.

Diversidad, igualdad, atención a la diversidad, igualdad de oportunidades, son conceptos muy utilizados en el discurso pedagógico al uso "políticamente correcto». Sin embargo, la mayor parte de las veces, las prácticas reales desarrolladas en los centros no son permeables a las implicaciones derivadas de asumir su significado. Los cambios introducidos para dar respuesta a la diversidad se centran en el ámbito de los recursos humanos y materiales $y$, alguna vez, en modificaciones de los procesos de enseñanza-aprendizaje. Estas medidas suelen ser puntuales y desarrolladas «en paralelo» al currículum ordinario con el consiguiente peligro de estigmatizar a determinados alumnos y colectivos colocados sistemáticamente en clases de compensatoria, de necesidades especiales, de integración, de refuerzo, ... 
Es preciso ejercer la práctica del discurso sobre igualdad y diversidad como parte del debate educativo, y hacerlo en el interior mismo de la escuela, en las aulas, claustros, comisiones, órganos de representación. Son muchas las resistencias a desarrollar los planteamientos de atención a la diversidad exigidos por la reforma, se manifiestan en dimensiones estructurales de los centros y en los procesos educativos, pero también en las opiniones y actitudes de los mismos profesionales e investigadores. La propuesta aquí planteada pasa por revisar los significados otorgados a la diversidad, contribuir a promover una cultura inclusiva en los centros y a desarrollar prácticas que garanticen el éxito académico a todos los grupos. Esta debería ser una exigencia irrenunciable en toda etapa educativa de carácter obligatorio.

\section{Referencias bibliográficas}

Aguado, M. T. (1995): «Investigación en Educación Multicultural: limitaciones y perspectivas». En Actas II Congreso Internacional de Educación Intercultural para la Paz. UNED/Ayuntamiento de Ceuta, 23-26 abril.

Aguado, M. T. (1996): Educación Multicultural. Su teoría y su práctica. Cuadernos de la UNED. UNED, Madrid.

Aguado, M. T. (1997): Aportaciones conceptuales y metodológicas en tres ámbitos de la pedagogía diferencial. Educación Intercultural. En Revista de Investigación Educativa, vol. 15, 2, 247-271.

Aguado, M. T. (1998): Diversidad cultural e igualdad escolar. Un modelo para el diagnóstico y desarrollo de actuaciones educativas en contextos escolares multiculturales. Serie Investigación. Madrid: CIDE/MEC, $\mathrm{n}^{\circ} 141$.

Aguado, M. T. (2000): Mediación social en el ámbito educativo. Cursos de Formación, documento inédito. Escuela de Mediadores Sociales para la Inmigración. Madrid: EMSI.

Banks, J. A. (1996): Multicultural Education: Historical Development, dimensions and practice. En Banks, J. A. y McGee Banks, Ch. A. (eds.): Handbook of Research on Multicultural Education. (pp. 3-23). New York: McMillan Pub.

Baptiste, H. P. (1999): The multicultural environment of schools: Implications to leaders. En L. W. Hughes, (ed.), The principal as leader, (pp. 105-127). Upper Saddle River, NJ: Merrill.

Bennett, Ch. (1990): Comprehensive Multicultural Education: Theory and Practice. New York: Allyn and Bacon.

COHEN y cols. (1968): Conceptual styles, culture conflict and noverbal test of intelligence. En American Anthropologist, 71, 828-856.

Coleman, J. S. (1966): Equality of educational Opportunity. National Center for Educational Statistic. Washington.

Corson, D. (1995): Discursive power in educational organizations: An introduction. En D. Corson (ed.), Discourse and power in educational organizations. (pp. 3-15) Cresskill, NJ: Hampton Press, Ins.

Fernández Enguita, M. (1995): Unidad y diversidad en la escuela comprensiva. En Cuadernos de Pedagogía, 172, 72-75.

Foucault, M. (1980): Power/knowledge. Selected interviews and other writings 1972-1977. Nueva York: Pantheon Books.

Giroux, H. A. (1991): Theory and Resistance in Education. Bergin and Garvey, South Hadley, MA. 
Hargreaves, A. (1994): Changing teachers, changing times: Teachers'work and culture in the postmodern age. Nueva York: Teachers College Press.

Jackson, G. y Cosca, C. (1974): «The inequality of educational opportunities in the Southwest. An ovservational study». En American Educational Research Journal, 11, 3, 219-229.

Katz, A. (1999): Keepin'it real: Personalizing school experiences for diverse learners to create hermony instead of conflict. Encuentro anual de la American Educational Association, Montreal, Canada. (paper).

Lluch i Balaguer, X. y Salinas Catalá, J. (1996): La diversidad cultural en la práctica educativa. Materiales para la formación del profesorado en educación intercultural. MEC, Madrid.

L.O.G.S.E. (1990): Ley de Ordenación General del Sistema Educativo. MEC, Madrid.

Miron, L. F. (1997): Resisting discrimination: Affirmative strategies for principals and teachers. Thousand Oaks, CA: Corwin Press, Inc.

Nieto, S. (1992): Affirming diversity. The sociopolitical context for Multicultural Education. Longman, New York.

Parker, L. y SHAPIRo, J. P. (1993): The context of educational administration and social class. En C.A. Capper (ed.), Educational practices in a pluralistic society. Albany: State University New York Press.

Pérez, A. (1995): Autonomía y formación para la diversidad. En Cuadernos de Pedagogía, 172, 5862.

Pérez, C. (1998): Metodología de atención a la diversidad en el aula. En Revista Española de Orientación y Psicopedagogía, vol. 9, 16, 273-287.

Rivera, M. (2000): La atención a lo singular en la relación educativa. En Cuadernos de Pedagogía. Monográfico sobre Atención a la diversidad, 293, 10-13.

Rosenthal, R. y Jackobson, W. (1968): Pygmalion on the classroom. Teachers expectations and pupils'intellectual performance. Holt, Rinehart and Winston, Nueva York.

Samuda, R. y Crawford, A. (1986): Multiculuralism in Canada. Social and Educational perspectives. Allyn and Bacon. Ontario.

Sánchez Valle, J. (1992): Diversidad cultural e investigación educativa. Universidad Complutense, Madrid.

Scanell, P., Schlesinger, P. y Sparks, C. (1992) (eds.): Culture and power. A media, culture and society reader. SAGE pub., Londres.

Skutnabb-Kangas, T. (1990): Legitimating or deligitimating new forms of racism. Role of researchers». En Journal of Multilingual and Multicultural Development, VII, 1 \& 2, 77-100.

Walker, E. W. (1999): Conflict in the house:Interethnic conflict as change agent, change as conflict instigator. Congreso Anual de la American Educational Research Association, Montreal, Canada. (paper)

Fecha de recepción: 30-10-00

Fecha de revisión: 15-11-00

Fecha de aceptación: 21-11-00 